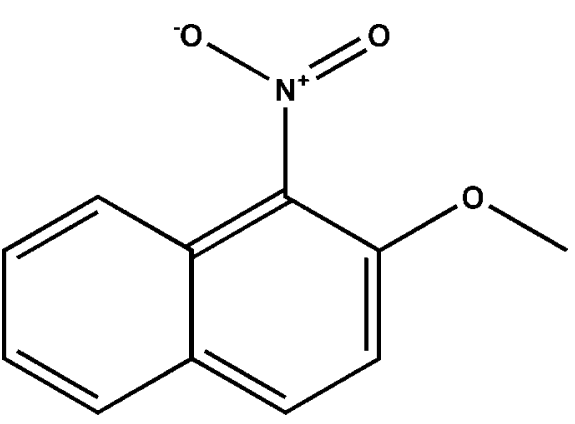

\title{
Crystal structure of 2-methoxy-1-nitro- naphthalene
}

\section{Hasna Yassine, ${ }^{a}$ Mostafa Khouili, ${ }^{a *}$ Lahcen El Ammari, ${ }^{b}$ Mohamed Saadi ${ }^{\mathrm{b}}$ and El Mostafa Ketatni ${ }^{\mathrm{c}}$}

aLaboratoire de Chimie Organique et Analytique, Université Sultan Moulay Slimane, Faculté des Sciences et Techniques, BP 523, 23000 Béni-Mellal, Morocco, baboratoire de Chimie du Solide Appliquée, Faculté des Sciences, Université Mohammed V, Avenue Ibn Battouta, BP 1014, Rabat, Morocco, and ' 'Laboratoire de Spectrochimie Applique et Environnement, Université Sultan Moulay Slimane, Faculté des Sciences et Techniques, BP 523, 23000 Béni-Mellal, Morocco.

*Correspondence e-mail: m.khouili@usms.ma

Received 2 August 2015; accepted 28 August 2015

Edited by W. T. A. Harrison, University of Aberdeen, Scotland

The asymmetric unit of the title compound, $\mathrm{C}_{11} \mathrm{H}_{9} \mathrm{NO}_{3}$, contains two molecules, $A$ and $B$. In molecule $A$, the dihedral angle between the planes of the naphthalene ring system (r.m.s. deviation $=0.003 \AA$ ) and the nitro group is $89.9(2)^{\circ}$, and the $\mathrm{C}$ atom of the methoxy group deviates from the naphthyl plane by 0.022 (2) A. Equivalent data for molecule $B$ are $0.008 \AA, 65.9(2)^{\circ}$ and -0.198 (2) $\AA$, respectively. In the crystal, molecules are linked by weak $\mathrm{C}-\mathrm{H} \cdots \mathrm{O}$ interactions, forming [100] chains of alternating $A$ and $B$ molecules. Weak aromatic $\pi-\pi$ stacking contacts, with a range of centroidcentroid distances from 3.5863 (9) to 3.8048 (9) $\AA$, are also observed.

Keywords: crystal structure; naphthalene derivative; weak $\mathrm{C}-\mathrm{H} \cdots \mathrm{O}$ interactions; $\pi-\pi$ stacking.

CCDC reference: 1421062

\section{Related literature}

For biological activities of naphthalene derivatives, see: Wright et al. (2000); Rokade \& Sayyed (2009); Upadhayaya et al. (2010). For the title compound as an intermediate in the synthesis of antipyretic drugs, see: Stoylkova et al. (2000); Govindarajana et al. (2011); Kirumakki et al. (2004); Yadav et al. (1998). For a related structure, see: Wannalerse et al. (2013).

\section{Experimental}

\subsection{Crystal data}

$\mathrm{C}_{11} \mathrm{H}_{9} \mathrm{NO}_{3}$

$M_{r}=203.19$

Triclinic, $P \overline{1}$

$a=9.1291$ (4) ^

$b=10.2456(4) \AA$

$c=10.5215$ (4) $\AA$

$\alpha=86.390(2)^{\circ}$

$\beta=82.964(2)^{\circ}$

\subsection{Data collection}

Bruker X8 APEXII CCD diffractometer

Absorption correction: multi-scan (SADABS; Bruker, 2009)

$T_{\min }=0.676, T_{\max }=0.746$

\subsection{Refinement}

$R\left[F^{2}>2 \sigma\left(F^{2}\right)\right]=0.048$

$w R\left(F^{2}\right)=0.141$

$S=1.04$

5450 reflections

$\gamma=85.801(2)^{\circ}$

$V=972.63(7) \AA^{3}$

$Z=4$

Mo $K \alpha$ radiation

$\mu=0.10 \mathrm{~mm}^{-1}$

$T=296 \mathrm{~K}$

$0.39 \times 0.32 \times 0.24 \mathrm{~mm}$

34901 measured reflections 5450 independent reflections 3446 reflections with $I>2 \sigma(I)$ $R_{\text {int }}=0.038$

Table 1

Hydrogen-bond geometry $\left(\AA{ }^{\circ}\right)$.

\begin{tabular}{lllll}
\hline$D-\mathrm{H} \cdots A$ & $D-\mathrm{H}$ & $\mathrm{H} \cdots A$ & $D \cdots A$ & $D-\mathrm{H} \cdots A$ \\
\hline $\mathrm{C} 4-\mathrm{H} 4 \cdots \mathrm{O}^{\mathrm{i}}$ & 0.93 & 2.57 & $3.409(2)$ & 150 \\
$\mathrm{C} 11-\mathrm{H} 11 A \cdots \mathrm{O}^{\mathrm{ii}}$ & 0.96 & 2.60 & $3.462(3)$ & 150 \\
\hline
\end{tabular}

Symmetry codes: (i) $x+1, y+1, z$; (ii) $x, y+1, z$.

Data collection: APEX2 (Bruker, 2009); cell refinement: SAINT (Bruker, 2009); data reduction: $S A I N T$; program(s) used to solve structure: SHELXS97 (Sheldrick, 2008); program(s) used to refine structure: SHELXL97 (Sheldrick, 2008); molecular graphics: ORTEP-3 for Windows (Farrugia, 2012); software used to prepare material for publication: PLATON (Spek, 2009) and publCIF (Westrip, 2010).

\section{Acknowledgements}

The authors thank the Unit of Support for Technical and Scientific Research (UATRS, CNRST) for the X-ray measurements and the University Sultan Moulay Slimane, Beni-Mellal, for financial support. 
Supporting information for this paper is available from the IUCr electronic archives (Reference: HB7477).

\section{References}

Bruker (2009). APEX2, SAINT and SADABS. Bruker AXS Inc., Madison, Wisconsin, USA.

Farrugia, L. J. (2012). J. Appl. Cryst. 45, 849-854.

Govindarajan, M., Ganasan, K., Periandy, S. \& Karabacak, M. (2011). Spectrochim. Acta A Mol. Biomol. Spectrosc. 79, 646-653.

Kirumakki, S. R., Nagaraju, N., Chary, K. V. R. \& Narayanan, S. (2004). J. Catal. 221, 549-559.
Rokade, Y. B. \& Sayyed, R. Z. (2009). Rasayan J. Chem. 2, 972-980

Sheldrick, G. M. (2008). Acta Cryst. A64, 112-122.

Spek, A. L. (2009). Acta Cryst. D65, 148-155.

Stoylkova, T. Y., Chanev, C. D., Lechert, H. T. \& Bezouhanova, C. P. (2000). Catal. Lett. 69, 109-112.

Upadhayaya, R. S., Vandavasi, J. K., Kardile, R. A., Lahore, S. V., Dixit, S. S., Deokar, H. S., Shinde, P. D., Sarmah, M. P. \& Chattopadhyaya, J. (2010). Eur. J. Med. Chem. 45, 1854-1867.

Wannalerse, B., Pannil, W., Loriang, J., Tuntulani, T. \& Duangthongyou, T. (2013). Acta Cryst. E69, o682.

Westrip, S. P. (2010). J. Appl. Cryst. 43, 920-925.

Wright, M. S., Lax, A. R., Henderson, G. \& Chen, J. A. (2000). Mycologia, 92, 42-45.

Yadav, G. D. \& Krishnan, S. (1998). Ind. Eng. Chem. Res. 37, 3358-3365. 


\section{supporting information}

Acta Cryst. (2015). E71, o701-o702［doi:10.1107/S2056989015016114]

\section{Crystal structure of 2-methoxy-1-nitronaphthalene}

\section{Hasna Yassine, Mostafa Khouili, Lahcen El Ammari, Mohamed Saadi and El Mostafa Ketatni}

\section{S1. Comment}

Naphthalene derivatives have been extensively employed in many fields, for example, as a colorant, explosive, disinfectant, insecticide, auxin plant hormone and play a role in the chemical defence against biological (Wright et al., 2000) and have diverse and interesting antibiotic properties (Rokade \& Sayyed, 2009; Upadhayaya et al. 2010). 2-Methoxynaphthalene is an important intermediate used in the production of naproxen. It is widely used a non-steroidal antiinflammatory, analgesic and antipyretic drug (Stoylkova et al., 2000, Govindarajana et al., 2011, Kirumakki et al., 2004; Yadav et al., 1998). Nitration of 1-methoxynaphthalene with bismuth nitrate in $\mathrm{CH}_{2} \mathrm{Cl}_{2}$ gives a compound (I) and describes its structure here.

The asymmetric unit of the title compound consists of two crystallographically independent molecules of nearly similar geometry as shown in Fig. 1. Bond lengths and angles of the title compound are comparable with that found in the similar structure (Wannalerse et al., 2013). In the first (O1O2O3N1C1-C11) and second (O4O5O6N2C12-C22) molecules, the dihedral angles between the nitro group and the attached naphthalene system are $89.9(2)^{\circ}$ and $65.9(2)^{\circ}$, respectively. The two naphthalene rings belonging to the both molecules form a dihedral angle of $72.02(5)^{\circ}$.

In the crystal, the molecules are linked together by weak $\mathrm{C}-\mathrm{H} \cdots \mathrm{O}$ interactions. Moreover, the $\pi-\pi$ contacts between the naphthalene rings, may further consolidate the structure, with range of centroid- centroid distances $=3.5863(9)-3.8048$ (9) $\AA$.

\section{S2. Experimental}

2-Methoxynaphtalene $(500 \mathrm{mg}, 3.164 \mathrm{mmol})$ and silica gel $(500 \mathrm{mg})$ was added to a suspension of bismuth nitrate pentahydrate (1.2 eqv.) in $\mathrm{CH}_{2} \mathrm{Cl}_{2}(20 \mathrm{ml})$. The mixture was refluxed for $6 \mathrm{~h}$. After cooling to room temperature, the reaction mixture was filtered and watched with $\mathrm{CH}_{2} \mathrm{Cl}_{2}$, the filtrate obtained was concentrated, and the resulting residue was purified by column chromatography using EtOAc-Hexane $(1: 9 \mathrm{v} / \mathrm{v})$. The title compound was recrystallized from the solvent mixture ethyl acetate/hexane to yield orange block crystals (yield: 74\%).

\section{S3. Refinement}

All $\mathrm{H}$ atoms could be located in a difference Fourier map. However, they were placed in calculated positions with $\mathrm{C}-\mathrm{H}$ $=0.93-0.96 \AA$, and refined as riding on their parent atoms with $U_{\text {iso }}(\mathrm{H})=1.2 U_{\text {eq }}$ for aromatic and $U_{\text {iso }}(\mathrm{H})=1.5 U_{\text {eq }}(C)$ for methyl. Two outlier reflections, (-7 30$)$ and (-1 - 32 2), were omitted in the last cycles of refinement. 

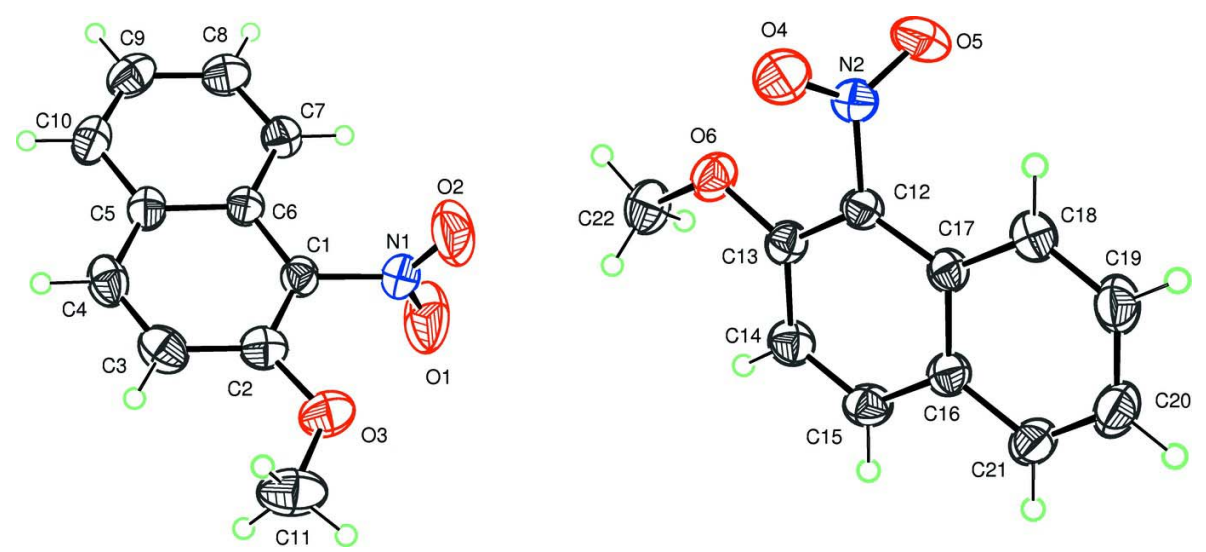

\section{Figure 1}

A view of the molecule of the title compound, showing displacement ellipsoids drawn at the $50 \%$ probability level. $\mathrm{H}$ atoms are represented as small circles.

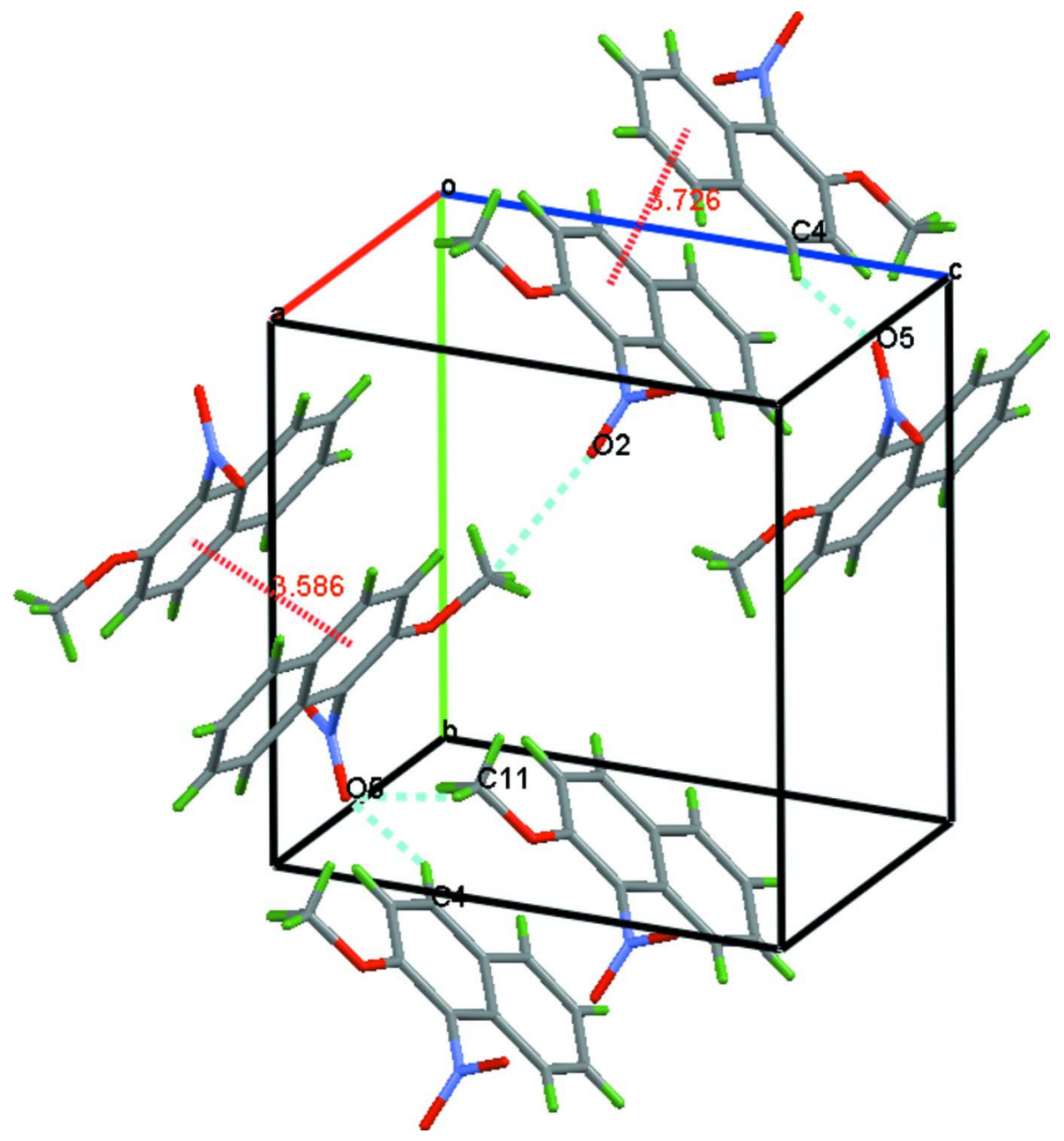

\section{Figure 2}

Partial crystal packing for the title compound showing molecules linked by hydrogen bonds as blue dashed lines and $\pi-\pi$ contacts between the naphthalene rings (red dashed lines). 


\section{2-Methoxy-1-nitronaphthalene}

Crystal data

$\mathrm{C}_{11} \mathrm{H}_{9} \mathrm{NO}_{3}$

$M_{r}=203.19$

Triclinic, $P \overline{1}$

$a=9.1291(4) \AA$

$b=10.2456$ (4) $\AA$

$c=10.5215(4) \AA$

$\alpha=86.390(2)^{\circ}$

$\beta=82.964(2)^{\circ}$

$\gamma=85.801(2)^{\circ}$

$V=972.63(7) \AA^{3}$

\section{Data collection}

\section{Bruker X8 APEXII CCD}

diffractometer

Radiation source: fine-focus sealed tube

Graphite monochromator

$\varphi$ and $\omega$ scans

Absorption correction: multi-scan

(SADABS; Bruker, 2009)

$T_{\min }=0.676, T_{\max }=0.746$

\section{Refinement}

Refinement on $F^{2}$

Least-squares matrix: full

$R\left[F^{2}>2 \sigma\left(F^{2}\right)\right]=0.048$

$w R\left(F^{2}\right)=0.141$

$S=1.04$

5450 reflections

272 parameters

0 restraints

Hydrogen site location: inferred from neighbouring sites
$Z=4$

$F(000)=424$

$D_{\mathrm{x}}=1.388 \mathrm{Mg} \mathrm{m}^{-3}$

Mo $K \alpha$ radiation, $\lambda=0.71073 \AA$

Cell parameters from 3506 reflections

$\theta=1.7-30.0^{\circ}$

$\mu=0.10 \mathrm{~mm}^{-1}$

$T=296 \mathrm{~K}$

Block, orange

$0.39 \times 0.32 \times 0.24 \mathrm{~mm}$

34901 measured reflections

5450 independent reflections

3446 reflections with $I>2 \sigma(I)$

$R_{\text {int }}=0.038$

$\theta_{\text {max }}=29.6^{\circ}, \theta_{\min }=2.0^{\circ}$

$h=-12 \rightarrow 12$

$k=-14 \rightarrow 14$

$l=-14 \rightarrow 14$

$\mathrm{H}$-atom parameters constrained

$w=1 /\left[\sigma^{2}\left(F_{\mathrm{o}}^{2}\right)+(0.0511 P)^{2}+0.2462 P\right]$

where $P=\left(F_{\mathrm{o}}{ }^{2}+2 F_{\mathrm{c}}{ }^{2}\right) / 3$

$(\Delta / \sigma)_{\max }<0.001$

$\Delta \rho_{\max }=0.20 \mathrm{e}^{-3}$

$\Delta \rho_{\min }=-0.15$ e $\AA^{-3}$

Extinction correction: SHELXL97 (Sheldrick, 2008), $\mathrm{Fc}^{*}=\mathrm{kFc}\left[1+0.001 \mathrm{xFc}^{2} \lambda^{3} / \sin (2 \theta)\right]^{-1 / 4}$

Extinction coefficient: 0.011 (2)

\section{Special details}

Geometry. All e.s.d.'s (except the e.s.d. in the dihedral angle between two 1.s. planes) are estimated using the full covariance matrix. The cell e.s.d.'s are taken into account individually in the estimation of e.s.d.'s in distances, angles and torsion angles; correlations between e.s.d.'s in cell parameters are only used when they are defined by crystal symmetry. An approximate (isotropic) treatment of cell e.s.d.'s is used for estimating e.s.d.'s involving 1.s. planes.

Fractional atomic coordinates and isotropic or equivalent isotropic displacement parameters $\left(\hat{A}^{2}\right)$

\begin{tabular}{lllll}
\hline & $x$ & $y$ & $z$ & $U_{\text {iso }} * / U_{\text {eq }}$ \\
\hline C1 & $0.82138(17)$ & $0.83303(15)$ & $0.59386(15)$ & $0.0445(3)$ \\
C2 & $0.76837(19)$ & $0.92960(16)$ & $0.67524(16)$ & $0.0512(4)$ \\
C3 & $0.8714(2)$ & $0.99956(17)$ & $0.72711(17)$ & $0.0582(4)$ \\
H3 & 0.8389 & 1.0650 & 0.7832 & $0.070^{*}$ \\
C4 & $1.0189(2)$ & $0.97177(17)$ & $0.69542(16)$ & $0.0562(4)$ \\
H4 & 1.0854 & 1.0201 & 0.7298 & $0.067^{*}$ \\
C5 & $1.07461(18)$ & $0.87266(15)$ & $0.61254(15)$ & $0.0464(4)$ \\
C6 & $0.97310(17)$ & $0.79982(14)$ & $0.55904(14)$ & $0.0416(3)$
\end{tabular}




\begin{tabular}{|c|c|c|c|c|}
\hline $\mathrm{C} 7$ & $1.02802(19)$ & $0.70018(16)$ & $0.47545(16)$ & $0.0515(4)$ \\
\hline $\mathrm{H} 7$ & 0.9625 & 0.6519 & 0.4398 & $0.062 *$ \\
\hline $\mathrm{C} 8$ & $1.1760(2)$ & $0.67452(19)$ & $0.44694(18)$ & $0.0605(5)$ \\
\hline H8 & 1.2107 & 0.6085 & 0.3920 & $0.073^{*}$ \\
\hline C9 & $1.2771(2)$ & $0.7456(2)$ & 0.49869 (19) & $0.0633(5)$ \\
\hline H9 & 1.3782 & 0.7266 & 0.4781 & $0.076^{*}$ \\
\hline $\mathrm{C} 10$ & $1.2280(2)$ & $0.84240(19)$ & $0.57914(18)$ & $0.0580(5)$ \\
\hline $\mathrm{H} 10$ & 1.2961 & 0.8895 & 0.6128 & $0.070^{*}$ \\
\hline $\mathrm{C} 11$ & $0.5602(3)$ & $1.0473(2)$ & $0.7849(2)$ & $0.0859(7)$ \\
\hline H11A & 0.4542 & 1.0518 & 0.7914 & $0.129^{*}$ \\
\hline H11B & 0.5951 & 1.1311 & 0.7543 & $0.129 *$ \\
\hline $\mathrm{H} 11 \mathrm{C}$ & 0.5912 & 1.0242 & 0.8678 & $0.129^{*}$ \\
\hline $\mathrm{C} 12$ & $0.12086(16)$ & $0.31177(13)$ & $0.90308(14)$ & $0.0391(3)$ \\
\hline $\mathrm{C} 13$ & $0.19472(16)$ & $0.40531(14)$ & $0.82703(14)$ & $0.0411(3)$ \\
\hline $\mathrm{C} 14$ & $0.11092(19)$ & $0.51331(15)$ & $0.77661(15)$ & $0.0477(4)$ \\
\hline $\mathrm{H} 14$ & 0.1581 & 0.5779 & 0.7242 & $0.057^{*}$ \\
\hline $\mathrm{C} 15$ & $-0.03834(18)$ & $0.52299(15)$ & $0.80456(15)$ & $0.0483(4)$ \\
\hline H15 & -0.0915 & 0.5959 & 0.7721 & $0.058^{*}$ \\
\hline $\mathrm{C} 16$ & $-0.11572(17)$ & $0.42663(14)$ & $0.88090(14)$ & $0.0424(3)$ \\
\hline $\mathrm{C} 17$ & $-0.03443(16)$ & $0.31586(14)$ & $0.93202(14)$ & $0.0391(3)$ \\
\hline $\mathrm{C} 18$ & -0.11305 (19) & $0.22006(16)$ & $1.00938(17)$ & $0.0515(4)$ \\
\hline H18 & -0.0618 & 0.1475 & 1.0446 & $0.062 *$ \\
\hline C19 & $-0.2632(2)$ & $0.23339(18)$ & $1.0326(2)$ & $0.0612(5)$ \\
\hline H19 & -0.3135 & 0.1690 & 1.0827 & $0.073^{*}$ \\
\hline $\mathrm{C} 20$ & -0.34344 (19) & $0.34223(19)$ & $0.9822(2)$ & $0.0618(5)$ \\
\hline $\mathrm{H} 20$ & -0.4460 & 0.3497 & 0.9990 & $0.074 *$ \\
\hline $\mathrm{C} 21$ & $-0.27129(18)$ & $0.43676(17)$ & $0.90895(18)$ & $0.0532(4)$ \\
\hline $\mathrm{H} 21$ & -0.3251 & 0.5093 & 0.8767 & $0.064^{*}$ \\
\hline $\mathrm{C} 22$ & $0.4201(2)$ & $0.47481(19)$ & $0.71352(18)$ & $0.0615(5)$ \\
\hline $\mathrm{H} 22 \mathrm{~A}$ & 0.5244 & 0.4516 & 0.7073 & $0.092 *$ \\
\hline $\mathrm{H} 22 \mathrm{~B}$ & 0.4010 & 0.5629 & 0.7403 & $0.092 *$ \\
\hline $\mathrm{H} 22 \mathrm{C}$ & 0.3862 & 0.4688 & 0.6314 & $0.092 *$ \\
\hline N1 & $0.20980(15)$ & $0.20198(13)$ & $0.95773(14)$ & $0.0483(3)$ \\
\hline N2 & $0.71338(16)$ & $0.76148(15)$ & $0.53852(16)$ & $0.0571(4)$ \\
\hline $\mathrm{O} 1$ & $0.6734(2)$ & $0.8025(2)$ & $0.43824(19)$ & $0.1118(7)$ \\
\hline $\mathrm{O} 2$ & $0.6708(2)$ & $0.66298(17)$ & $0.5941(2)$ & $0.1043(6)$ \\
\hline $\mathrm{O} 3$ & $0.61970(14)$ & $0.95078(14)$ & $0.69777(14)$ & $0.0703(4)$ \\
\hline $\mathrm{O} 4$ & $0.29054(17)$ & $0.22522(13)$ & $1.03577(15)$ & $0.0785(4)$ \\
\hline O5 & $0.19774(17)$ & $0.09259(12)$ & $0.92409(16)$ & $0.0767(4)$ \\
\hline O6 & $0.34371(12)$ & $0.38730(11)$ & $0.80514(11)$ & $0.0537(3)$ \\
\hline
\end{tabular}

Atomic displacement parameters $\left(\AA^{2}\right)$

\begin{tabular}{lllllll}
\hline & $U^{11}$ & $U^{22}$ & $U^{33}$ & $U^{12}$ & $U^{13}$ & $U^{23}$ \\
\hline C1 & $0.0446(8)$ & $0.0428(8)$ & $0.0464(8)$ & $-0.0077(6)$ & $-0.0072(7)$ & $0.0025(6)$ \\
C2 & $0.0539(10)$ & $0.0513(9)$ & $0.0462(9)$ & $-0.0005(7)$ & $-0.0013(7)$ & $0.0027(7)$ \\
C3 & $0.0772(13)$ & $0.0527(9)$ & $0.0447(9)$ & $-0.0034(9)$ & $-0.0046(9)$ & $-0.0084(7)$ \\
C4 & $0.0708(12)$ & $0.0557(10)$ & $0.0465(9)$ & $-0.0177(8)$ & $-0.0179(8)$ & $-0.0006(7)$
\end{tabular}




\begin{tabular}{lllllll} 
C5 & $0.0510(9)$ & $0.0486(8)$ & $0.0410(8)$ & $-0.0110(7)$ & $-0.0117(7)$ & $0.0069(7)$ \\
C6 & $0.0441(8)$ & $0.0415(7)$ & $0.0393(8)$ & $-0.0064(6)$ & $-0.0076(6)$ & $0.0052(6)$ \\
C7 & $0.0527(10)$ & $0.0499(9)$ & $0.0526(10)$ & $-0.0041(7)$ & $-0.0081(8)$ & $-0.0046(7)$ \\
C8 & $0.0582(11)$ & $0.0624(11)$ & $0.0580(11)$ & $0.0062(8)$ & $-0.0016(8)$ & $-0.0027(8)$ \\
C9 & $0.0455(10)$ & $0.0768(13)$ & $0.0640(12)$ & $0.0008(9)$ & $-0.0028(8)$ & $0.0118(10)$ \\
C10 & $0.0499(10)$ & $0.0688(11)$ & $0.0578(11)$ & $-0.0170(8)$ & $-0.0169(8)$ & $0.0122(9)$ \\
C11 & $0.0839(16)$ & $0.0823(15)$ & $0.0800(15)$ & $0.0233(12)$ & $0.0198(12)$ & $-0.0064(12)$ \\
C12 & $0.0404(8)$ & $0.0360(7)$ & $0.0417(8)$ & $-0.0007(6)$ & $-0.0080(6)$ & $-0.0039(6)$ \\
C13 & $0.0390(8)$ & $0.0445(8)$ & $0.0403(8)$ & $-0.0033(6)$ & $-0.0047(6)$ & $-0.0046(6)$ \\
C14 & $0.0524(9)$ & $0.0456(8)$ & $0.0442(8)$ & $-0.0048(7)$ & $-0.0055(7)$ & $0.0049(6)$ \\
C15 & $0.0522(9)$ & $0.0444(8)$ & $0.0483(9)$ & $0.0032(7)$ & $-0.0121(7)$ & $0.0036(7)$ \\
C16 & $0.0413(8)$ & $0.0445(8)$ & $0.0431(8)$ & $-0.0009(6)$ & $-0.0099(6)$ & $-0.0089(6)$ \\
C17 & $0.0392(8)$ & $0.0386(7)$ & $0.0409(8)$ & $-0.0042(6)$ & $-0.0070(6)$ & $-0.0069(6)$ \\
C18 & $0.0493(9)$ & $0.0441(8)$ & $0.0611(10)$ & $-0.0087(7)$ & $-0.0048(8)$ & $0.0001(7)$ \\
C19 & $0.0505(10)$ & $0.0582(10)$ & $0.0746(12)$ & $-0.0188(8)$ & $0.0041(9)$ & $-0.0067(9)$ \\
C20 & $0.0370(9)$ & $0.0710(12)$ & $0.0785(13)$ & $-0.0071(8)$ & $-0.0011(8)$ & $-0.0203(10)$ \\
C21 & $0.0411(9)$ & $0.0570(10)$ & $0.0636(11)$ & $0.0021(7)$ & $-0.0125(8)$ & $-0.0125(8)$ \\
C22 & $0.0487(10)$ & $0.0745(12)$ & $0.0592(11)$ & $-0.0146(9)$ & $0.0053(8)$ & $0.0020(9)$ \\
N1 & $0.0435(7)$ & $0.0412(7)$ & $0.0595(8)$ & $-0.0011(5)$ & $-0.0071(6)$ & $0.0016(6)$ \\
N2 & $0.0450(8)$ & $0.0572(9)$ & $0.0709(10)$ & $-0.0085(6)$ & $-0.0111(7)$ & $-0.0039(7)$ \\
O1 & $0.1262(16)$ & $0.1253(15)$ & $0.1010(13)$ & $-0.0526(12)$ & $-0.0681(12)$ & $0.0190(11)$ \\
O2 & $0.1055(13)$ & $0.0811(11)$ & $0.1351(16)$ & $-0.0523(10)$ & $-0.0365(11)$ & $0.0232(10)$ \\
O3 & $0.0542(8)$ & $0.0768(9)$ & $0.0753(9)$ & $0.0099(6)$ & $0.0049(6)$ & $-0.0104(7)$ \\
O4 & $0.0826(10)$ & $0.0663(8)$ & $0.0944(11)$ & $-0.0031(7)$ & $-0.0500(9)$ & $0.0107(7)$ \\
O5 & $0.0839(10)$ & $0.0403(7)$ & $0.1082(12)$ & $0.0091(6)$ & $-0.0230(8)$ & $-0.0139(7)$ \\
O6 & $0.0394(6)$ & $0.0609(7)$ & $0.0585(7)$ & $-0.0050(5)$ & $-0.0002(5)$ & $0.0050(5)$ \\
& & & & & & \\
\hline & & & & & &
\end{tabular}

Geometric parameters $\left(\AA,{ }^{\circ}\right)$

\begin{tabular}{llll}
\hline $\mathrm{C} 1-\mathrm{C} 2$ & $1.372(2)$ & $\mathrm{C} 12-\mathrm{N} 1$ & $1.4678(19)$ \\
$\mathrm{C} 1-\mathrm{C} 6$ & $1.411(2)$ & $\mathrm{C} 13-\mathrm{O} 6$ & $1.3524(18)$ \\
$\mathrm{C} 1-\mathrm{N} 2$ & $1.466(2)$ & $\mathrm{C} 13-\mathrm{C} 14$ & $1.412(2)$ \\
$\mathrm{C} 2-\mathrm{O} 3$ & $1.353(2)$ & $\mathrm{C} 14-\mathrm{C} 15$ & $1.356(2)$ \\
$\mathrm{C} 2-\mathrm{C} 3$ & $1.404(3)$ & $\mathrm{C} 14-\mathrm{H} 14$ & 0.9300 \\
$\mathrm{C} 3-\mathrm{C} 4$ & $1.360(3)$ & $\mathrm{C} 15-\mathrm{C} 16$ & $1.408(2)$ \\
$\mathrm{C} 3-\mathrm{H} 3$ & 0.9300 & $\mathrm{C} 15-\mathrm{H} 15$ & 0.9300 \\
$\mathrm{C} 4-\mathrm{C} 5$ & $1.406(2)$ & $\mathrm{C} 16-\mathrm{C} 21$ & $1.412(2)$ \\
$\mathrm{C} 4-\mathrm{H} 4$ & 0.9300 & $\mathrm{C} 16-\mathrm{C} 17$ & $1.423(2)$ \\
$\mathrm{C} 5-\mathrm{C} 10$ & $1.418(2)$ & $\mathrm{C} 17-\mathrm{C} 18$ & $1.414(2)$ \\
$\mathrm{C} 5-\mathrm{C} 6$ & $1.419(2)$ & $\mathrm{C} 18-\mathrm{C} 19$ & $1.361(2)$ \\
$\mathrm{C} 6-\mathrm{C} 7$ & $1.413(2)$ & $\mathrm{C} 18-\mathrm{H} 18$ & 0.9300 \\
$\mathrm{C} 7-\mathrm{C} 8$ & $1.358(2)$ & $\mathrm{C} 19-\mathrm{C} 20$ & $1.400(3)$ \\
$\mathrm{C} 7-\mathrm{H} 7$ & 0.9300 & $\mathrm{C} 19-\mathrm{H} 19$ & 0.9300 \\
$\mathrm{C} 8-\mathrm{C} 9$ & $1.396(3)$ & $\mathrm{C} 20-\mathrm{C} 21$ & $1.357(3)$ \\
$\mathrm{C} 8-\mathrm{H} 8$ & 0.9300 & $\mathrm{C} 20-\mathrm{H} 20$ & 0.9300 \\
$\mathrm{C} 9-\mathrm{C} 10$ & $1.360(3)$ & $\mathrm{C} 21-\mathrm{H} 21$ & 0.9300 \\
$\mathrm{C} 9-\mathrm{H} 9$ & 0.9300 & $\mathrm{C} 22-\mathrm{O} 6$ & $1.427(2)$ \\
$\mathrm{C} 10-\mathrm{H} 10$ & 0.9300 & $\mathrm{C} 22-\mathrm{H} 22 \mathrm{~A}$ & 0.9600
\end{tabular}




\begin{tabular}{|c|c|c|c|}
\hline $\mathrm{C} 11-\mathrm{O} 3$ & $1.424(2)$ & $\mathrm{C} 22-\mathrm{H} 22 \mathrm{~B}$ & 0.9600 \\
\hline $\mathrm{C} 11-\mathrm{H} 11 \mathrm{~A}$ & 0.9600 & $\mathrm{C} 22-\mathrm{H} 22 \mathrm{C}$ & 0.9600 \\
\hline $\mathrm{C} 11-\mathrm{H} 11 \mathrm{~B}$ & 0.9600 & $\mathrm{~N} 1-\mathrm{O} 5$ & $1.2129(17)$ \\
\hline $\mathrm{C} 11-\mathrm{H} 11 \mathrm{C}$ & 0.9600 & $\mathrm{~N} 1-\mathrm{O} 4$ & $1.2136(18)$ \\
\hline $\mathrm{C} 12-\mathrm{C} 13$ & $1.371(2)$ & $\mathrm{N} 2-\mathrm{O} 1$ & $1.200(2)$ \\
\hline $\mathrm{C} 12-\mathrm{C} 17$ & $1.411(2)$ & $\mathrm{N} 2-\mathrm{O} 2$ & $1.200(2)$ \\
\hline $\mathrm{C} 2-\mathrm{C} 1-\mathrm{C} 6$ & $124.01(15)$ & $\mathrm{O} 6-\mathrm{C} 13-\mathrm{C} 14$ & $124.40(14)$ \\
\hline $\mathrm{C} 2-\mathrm{C} 1-\mathrm{N} 2$ & $117.77(15)$ & $\mathrm{C} 12-\mathrm{C} 13-\mathrm{C} 14$ & $118.16(14)$ \\
\hline $\mathrm{C} 6-\mathrm{C} 1-\mathrm{N} 2$ & $118.21(14)$ & $\mathrm{C} 15-\mathrm{C} 14-\mathrm{C} 13$ & $120.05(14)$ \\
\hline $\mathrm{O} 3-\mathrm{C} 2-\mathrm{C} 1$ & $116.87(16)$ & $\mathrm{C} 15-\mathrm{C} 14-\mathrm{H} 14$ & 120.0 \\
\hline $\mathrm{O} 3-\mathrm{C} 2-\mathrm{C} 3$ & $125.16(16)$ & $\mathrm{C} 13-\mathrm{C} 14-\mathrm{H} 14$ & 120.0 \\
\hline $\mathrm{C} 1-\mathrm{C} 2-\mathrm{C} 3$ & $117.96(16)$ & $\mathrm{C} 14-\mathrm{C} 15-\mathrm{C} 16$ & $122.34(14)$ \\
\hline $\mathrm{C} 4-\mathrm{C} 3-\mathrm{C} 2$ & $120.12(16)$ & $\mathrm{C} 14-\mathrm{C} 15-\mathrm{H} 15$ & 118.8 \\
\hline $\mathrm{C} 4-\mathrm{C} 3-\mathrm{H} 3$ & 119.9 & $\mathrm{C} 16-\mathrm{C} 15-\mathrm{H} 15$ & 118.8 \\
\hline $\mathrm{C} 2-\mathrm{C} 3-\mathrm{H} 3$ & 119.9 & $\mathrm{C} 15-\mathrm{C} 16-\mathrm{C} 21$ & $122.11(14)$ \\
\hline $\mathrm{C} 3-\mathrm{C} 4-\mathrm{C} 5$ & $122.44(16)$ & $\mathrm{C} 15-\mathrm{C} 16-\mathrm{C} 17$ & $118.92(14)$ \\
\hline $\mathrm{C} 3-\mathrm{C} 4-\mathrm{H} 4$ & 118.8 & $\mathrm{C} 21-\mathrm{C} 16-\mathrm{C} 17$ & $118.97(14)$ \\
\hline $\mathrm{C} 5-\mathrm{C} 4-\mathrm{H} 4$ & 118.8 & $\mathrm{C} 12-\mathrm{C} 17-\mathrm{C} 18$ & $124.80(14)$ \\
\hline $\mathrm{C} 4-\mathrm{C} 5-\mathrm{C} 10$ & $123.00(16)$ & $\mathrm{C} 12-\mathrm{C} 17-\mathrm{C} 16$ & $116.68(13)$ \\
\hline $\mathrm{C} 4-\mathrm{C} 5-\mathrm{C} 6$ & $118.74(15)$ & $\mathrm{C} 18-\mathrm{C} 17-\mathrm{C} 16$ & $118.51(14)$ \\
\hline $\mathrm{C} 10-\mathrm{C} 5-\mathrm{C} 6$ & $118.27(16)$ & $\mathrm{C} 19-\mathrm{C} 18-\mathrm{C} 17$ & $120.38(16)$ \\
\hline $\mathrm{C} 1-\mathrm{C} 6-\mathrm{C} 7$ & $124.13(14)$ & $\mathrm{C} 19-\mathrm{C} 18-\mathrm{H} 18$ & 119.8 \\
\hline $\mathrm{C} 1-\mathrm{C} 6-\mathrm{C} 5$ & $116.72(14)$ & $\mathrm{C} 17-\mathrm{C} 18-\mathrm{H} 18$ & 119.8 \\
\hline $\mathrm{C} 7-\mathrm{C} 6-\mathrm{C} 5$ & $119.15(15)$ & $\mathrm{C} 18-\mathrm{C} 19-\mathrm{C} 20$ & $121.21(17)$ \\
\hline $\mathrm{C} 8-\mathrm{C} 7-\mathrm{C} 6$ & $120.33(16)$ & $\mathrm{C} 18-\mathrm{C} 19-\mathrm{H} 19$ & 119.4 \\
\hline $\mathrm{C} 8-\mathrm{C} 7-\mathrm{H} 7$ & 119.8 & $\mathrm{C} 20-\mathrm{C} 19-\mathrm{H} 19$ & 119.4 \\
\hline $\mathrm{C} 6-\mathrm{C} 7-\mathrm{H} 7$ & 119.8 & $\mathrm{C} 21-\mathrm{C} 20-\mathrm{C} 19$ & $119.91(16)$ \\
\hline $\mathrm{C} 7-\mathrm{C} 8-\mathrm{C} 9$ & $121.14(18)$ & $\mathrm{C} 21-\mathrm{C} 20-\mathrm{H} 20$ & 120.0 \\
\hline $\mathrm{C} 7-\mathrm{C} 8-\mathrm{H} 8$ & 119.4 & $\mathrm{C} 19-\mathrm{C} 20-\mathrm{H} 20$ & 120.0 \\
\hline $\mathrm{C} 9-\mathrm{C} 8-\mathrm{H} 8$ & 119.4 & $\mathrm{C} 20-\mathrm{C} 21-\mathrm{C} 16$ & $121.01(16)$ \\
\hline $\mathrm{C} 10-\mathrm{C} 9-\mathrm{C} 8$ & $120.03(18)$ & $\mathrm{C} 20-\mathrm{C} 21-\mathrm{H} 21$ & 119.5 \\
\hline $\mathrm{C} 10-\mathrm{C} 9-\mathrm{H} 9$ & 120.0 & $\mathrm{C} 16-\mathrm{C} 21-\mathrm{H} 21$ & 119.5 \\
\hline $\mathrm{C} 8-\mathrm{C} 9-\mathrm{H} 9$ & 120.0 & $\mathrm{O} 6-\mathrm{C} 22-\mathrm{H} 22 \mathrm{~A}$ & 109.5 \\
\hline $\mathrm{C} 9-\mathrm{C} 10-\mathrm{C} 5$ & $121.09(17)$ & $\mathrm{O} 6-\mathrm{C} 22-\mathrm{H} 22 \mathrm{~B}$ & 109.5 \\
\hline $\mathrm{C} 9-\mathrm{C} 10-\mathrm{H} 10$ & 119.5 & $\mathrm{H} 22 \mathrm{~A}-\mathrm{C} 22-\mathrm{H} 22 \mathrm{~B}$ & 109.5 \\
\hline $\mathrm{C} 5-\mathrm{C} 10-\mathrm{H} 10$ & 119.5 & $\mathrm{O} 6-\mathrm{C} 22-\mathrm{H} 22 \mathrm{C}$ & 109.5 \\
\hline $\mathrm{O} 3-\mathrm{C} 11-\mathrm{H} 11 \mathrm{~A}$ & 109.5 & $\mathrm{H} 22 \mathrm{~A}-\mathrm{C} 22-\mathrm{H} 22 \mathrm{C}$ & 109.5 \\
\hline $\mathrm{O} 3-\mathrm{C} 11-\mathrm{H} 11 \mathrm{~B}$ & 109.5 & $\mathrm{H} 22 \mathrm{~B}-\mathrm{C} 22-\mathrm{H} 22 \mathrm{C}$ & 109.5 \\
\hline $\mathrm{H} 11 \mathrm{~A}-\mathrm{C} 11-\mathrm{H} 11 \mathrm{~B}$ & 109.5 & $\mathrm{O} 5-\mathrm{N} 1-\mathrm{O} 4$ & $123.26(14)$ \\
\hline $\mathrm{O} 3-\mathrm{C} 11-\mathrm{H} 11 \mathrm{C}$ & 109.5 & $\mathrm{O} 5-\mathrm{N} 1-\mathrm{C} 12$ & 118.58 \\
\hline $\mathrm{H} 11 \mathrm{~A}-\mathrm{C} 11-\mathrm{H} 11 \mathrm{C}$ & 109.5 & $\mathrm{O} 4-\mathrm{N} 1-\mathrm{C} 12$ & $118.15(13)$ \\
\hline $\mathrm{H} 11 \mathrm{~B}-\mathrm{C} 11-\mathrm{H} 11 \mathrm{C}$ & 109.5 & $\mathrm{O} 1-\mathrm{N} 2-\mathrm{O} 2$ & $122.74(17)$ \\
\hline $\mathrm{C} 13-\mathrm{C} 12-\mathrm{C} 17$ & $123.81(13)$ & $\mathrm{O} 1-\mathrm{N} 2-\mathrm{C} 1$ & $118.59(15)$ \\
\hline $\mathrm{C} 13-\mathrm{C} 12-\mathrm{N} 1$ & $117.52(13)$ & $\mathrm{O} 2-\mathrm{N} 2-\mathrm{C} 1$ & $118.65(16)$ \\
\hline $\mathrm{C} 17-\mathrm{C} 12-\mathrm{N} 1$ & $118.66(13)$ & $\mathrm{C} 2-\mathrm{O} 3-\mathrm{C} 11$ & $118.58(17)$ \\
\hline $\mathrm{O} 6-\mathrm{C} 13-\mathrm{C} 12$ & $117.43(13)$ & $\mathrm{C} 13-\mathrm{O} 6-\mathrm{C} 22$ & 117.88 \\
\hline
\end{tabular}




\section{supporting information}

Hydrogen-bond geometry $\left(A,{ }^{\circ}\right)$

\begin{tabular}{lllll}
\hline$D-\mathrm{H}^{\prime} \cdots A$ & $D-\mathrm{H}$ & $\mathrm{H} \cdots A$ & $D \cdots A$ & $D-\mathrm{H}^{\cdots} A A$ \\
\hline $\mathrm{C} 4-\mathrm{H} 4 \cdots \mathrm{O}^{\mathrm{i}}$ & 0.93 & 2.57 & $3.409(2)$ & 150 \\
$\mathrm{C} 11-\mathrm{H} 11 A \cdots \mathrm{O}^{\mathrm{ii}}$ & 0.96 & 2.60 & $3.462(3)$ & 150 \\
\hline
\end{tabular}

Symmetry codes: (i) $x+1, y+1, z$; (ii) $x, y+1, z$. 